\title{
An approach for optimizing gold nanoparticles for possible medical applications, using correlative electron energy loss and Raman spectroscopies on electron beam lithographically fabricated arrays
}

\author{
Robert Sinclair ${ }^{1, a)}$, Yitian Zeng ${ }^{1}\left(\mathbb{D}\right.$, Steven J. Madsen ${ }^{1}$, Sanjiv S. Gambhir ${ }^{2, b)}$ \\ ${ }^{1}$ Materials Science and Engineering, Stanford University, Stanford, CA 94305, USA \\ ${ }^{2}$ Molecular Imaging Program at Stanford, Canary Center at Stanford for Cancer Early Detection, Department of Radiology, Stanford University, \\ Stanford, CA 94305, USA \\ a) Address all correspondence to this author. e-mail: bobsinc@stanford.edu \\ ${ }^{b)}$ Deceased: Sanjiv S. Gambhir. \\ Received: 12 February 2021; accepted: 28 July 2021; published online: 24 September 2021
}

Surface-enhanced Raman spectroscopy (SERS), as induced by noble metal nanoparticles, is used for medical applications. In this study, gold nanoparticle parameters such as size, shape, separation, substrate etc. are varied systematically using electron beam lithographic fabrication methods. The resultant Raman spectra intensities are correlated with the nanoparticle surface plasmon energies as determined by electron energy loss spectroscopy (EELS) in a scanning transmission electron microscope (STEM) at nanometer-scale resolution. It is found that the largest enhancement is achieved when the illuminating laser energy closely matches, or is slightly higher than, the surface plasmon energies. In general, nanoparticle size is a strong determinant factor, and higher intensities are generated by sharply defined features. This approach allows a procedure to identify the optimum combination of nanoparticle parameters and laser energy to generate the largest Raman signals, thus enabling detection, for instance, of smaller, early-stage cancer tumors.

\section{Introduction}

There are numerous approaches to using "materials nanotechnology" for possible medical applications, including early cancer diagnosis [1-6]. Many rely on the detection of some sensitive physical property, such as magnetic, acoustic, or light signals [7-9] from nanoparticles attached to tumor cells, rather than the direct identification of the cells themselves. Oftentimes there is no overarching scientific methodology to optimize the nanoparticles other than by finding acceptably large signals either experimentally or by modeling. In this article, we describe procedures using lithographic techniques to systematically vary gold nanoparticle parameters such as size, shape, separation etc. to produce the highest intensity surface-enhanced Raman spectra (SERS), correlating the experimental observations with nanoparticle surface plasmon energies as determined by electron energy loss spectroscopy (EELS) in a transmission electron microscope (TEM).
The SERS phenomenon involves a very large increase in a normally weak laser-induced Raman signal from an organic dye, often by several orders of magnitude, induced by its contact with noble metal surfaces such as gold and silver $[10,11]$. This increase is known to be brought about by interaction with surface plasmons $[12,13]$. The effect has been utilized in several ways [13-15] in our research most notably for detection of cancer tumors utilizing silica-coated, $60 \mathrm{~nm}$ spherical gold nanoparticles containing Raman reporter molecules [16-18]. This has resulted in the development of an endoscope designed to illuminate, using a laser, the Raman signal from these particles potentially attached biochemically to small tumors within the bowel cavity $[19,20]$.

Various nanoparticle parameters are known to influence the intensity of the enhanced Raman signal such as size and shape [21-24]. In our electron microscopy studies of the particles attached to tumor cells, or to organs within a research animal, it 
is found that they often cluster $[22,25,26,17,27,28]$ (an example is shown in Supplementary Fig. S1 [29]) and so inter-particle separation and interaction is also possibly important. The type of dye and the particle composition are influential (but which are not considered in this work), as well as the metal particle dielectric coating which protects it from the environment in which it is operating. Varying all these in a controlled fashion to determine their individual effects is not straightforward and so we have applied lithographic fabrication methods in an attempt to do this systematically.

As SERS is associated with surface noble metal plasmons, the extensive research into plasmonic effects is relevant [30]. While photonic excitation of plasmons is highly effective [12, 31 ], it is more applicable to ensemble measurements with the limited resolution offered by light optical systems. Plasmons may also be induced by traveling electrons. Since the pioneering study [32] of plasmons in silver nanoprisms using electron energy loss spectroscopy in a scanning transmission electron microscope (STEM-EELS), this way of determining plasmon energies, showing their distribution within nanoparticles and the influence of size, shape, and separation (e.g., [33-37]), has become an important approach which we utilize here.

The present work, therefore, investigates the influence of nanoparticle parameters on the intensity of SERS signals and correlates these with surface plasmon energies and distributions using STEM-EELS and associated spectrum imaging on the exact same particles. Electron beam nanofabrication is applied to deposited polycrystalline gold thin films to create a range of particle sizes and spacings in the same sample, and initial studies are carried out on different shapes. As dielectric or metallic substrate environments can affect plasmon energies and amplitudes $[22,38,39]$, we utilize different substrates to establish their role, which provides a guide for suitable particle coating materials. Finally, as light-induced plasmon excitation is influenced by the illuminating photon energy [40], we also study the effects of different laser sources on Raman intensity, as correlated with possible resonance effects with the nanoparticle plasmon energies determined by STEM-EELS. This combined approach allows a procedure to systematically evaluate nanoparticle parameters which lead to the highest Raman signal, for the purpose of its application particularly in early cancer detection methodologies.

\section{Results}

\section{Raman spectroscopy on bulk substrate}

Arrays of 30-nm-thick, disk-shaped gold nanoparticles in $2 \times 2$ $\mu \mathrm{m}$ square segments were fabricated first on silicon substrates using electron beam lithography, as described in Experimental Details. Their diameters were varied from 20 to $200 \mathrm{~nm}$ in $5 \mathrm{~nm}$ increments along one axis, and their separation from 20 to 100 $\mathrm{nm}$ edge-to-edge along its perpendicular axis (See Supplementary Fig. S2 for scanning electron microscopy (SEM) images). Following characterization of an array by SEM, the structure was then coated with thiophenol Raman dye $(0.1 \mathrm{mg} / \mathrm{mL})$ and the variation of Raman spectra established in a Horiba LabRam confocal Raman microscope.

Figure 1a shows a typical result whereby it can be seen that maximum Raman intensity occurs for nanodisks in the 70 to $75 \mathrm{~nm}$ size range and that inter-particle separation has a lesser effect. Spectra from individual $2 \mu \mathrm{m}$ square boxes are inset in Fig. 1a.

An equivalent experiment carried out for nano "bow-tie" shapes (see Supplementary Fig. S3 for parameter definitions) yielded similar results (Fig. 1b), with maximum Raman signal in the 100 to $110 \mathrm{~nm}$ size range, with bow-tie separation having only a secondary influence. These results clearly show that the present lithographic approach provides an efficient method for testing the role that nanoparticle parameters can play in determining the intensity of SERS signals.

Because of the difficulty of subsequently making electron transparent specimens for detailed EELS analysis on specific array boxes, the e-beam lithographic procedures were adapted using thin film substrates attached to TEM grids, which is known to be a more difficult procedure [41]. This also allowed variation of the underlying dielectric material (which could then be employed as a protective coating for the nanoparticles), such as silica, silicon nitride, and crystalline silicon. Due to the size limitation of the transparent window aperture of the TEM grid, a subset of nanostructures was recreated on TEM grids (details are included in Supplementary Fig. S4). Raman spectra were collected after the electron microscopy, co-registered by the same grid position, and results for individual boxes are compared with the EELS data in the next section.

\section{STEM-EELS and Raman data on nanodisks}

Nanodisk STEM-EELS data were collected from 55, 75, 90, and $150 \mathrm{~nm}$ sized arrays at a constant inter-spacing of $75 \mathrm{~nm}$. Likewise, STEM-EELS data for $75 \mathrm{~nm}$ diameter disks were obtained for $35,75,90,110$, and $150 \mathrm{~nm}$ separation. Bow-tie EEL spectra were measured from 70,100 , and $140 \mathrm{~nm}$ sized arrays at a constant separation of $20 \mathrm{~nm}$, as well as 20 and $100 \mathrm{~nm}$ separated arrays at a constant size of $100 \mathrm{~nm}$ (see "STEM-EELS and Raman data on bow-tie nanoparticles" section). All arrays surveyed in the STEM-EELS are a subset of the whole array, scanning 100 by 100 pixels (2-4 nm/pixel) within individual boxes. This allowed subsequent analysis of the individual or collective EEL spectra as a function of nanoparticle parameters. Additionally, EEL spectra around nanodisk perimeters were also obtained from various sized particles ranging from $50-200 \mathrm{~nm}$ at a constant separation of $75 \mathrm{~nm}$. 
(a)

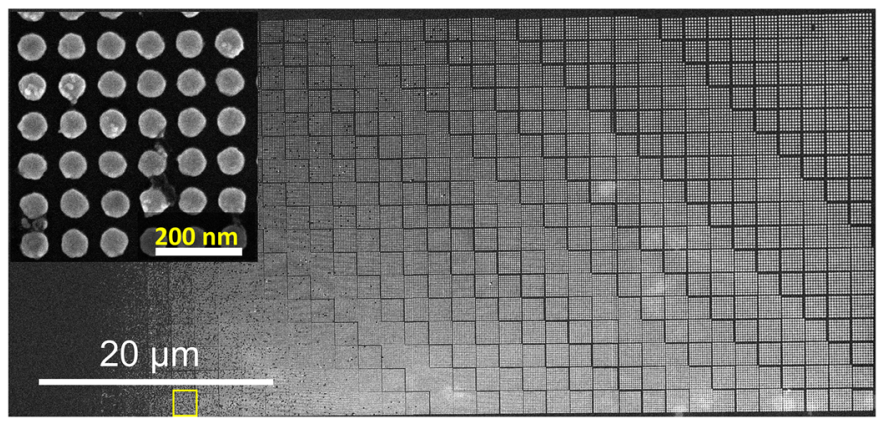

(b)

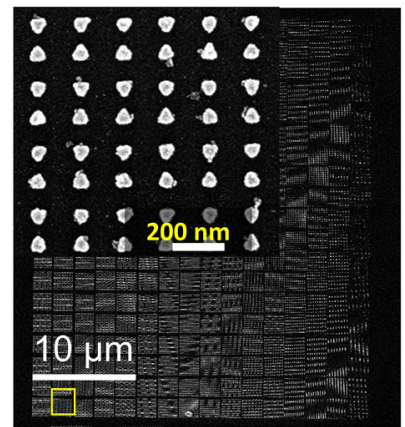

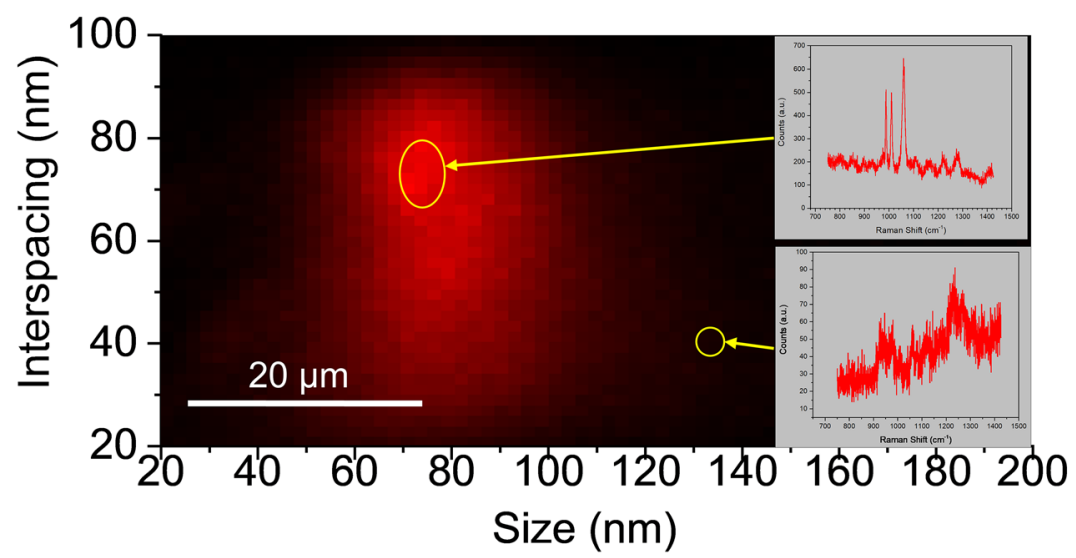

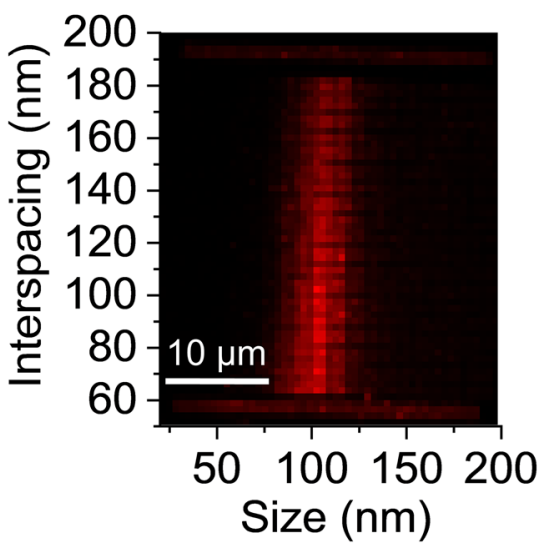

Figure 1: (a) SEM image (upper panel) and Raman map (lower panel) of nanodisk arrays with diameters ranging from 20 to $200 \mathrm{~nm}$ in $5 \mathrm{~nm}$ increments along the horizontal axis, and edge-to-edge inter-particle spacing from 20 to $100 \mathrm{~nm}$ along the vertical axis. The upper panel inset is an enlarged SEM image of the boxed region. The Raman map pixel intensity of each array configuration corresponds to the characteristic Raman peak intensity of thiophenol at $1060 \mathrm{~cm}^{-1}$ measured using a $785 \mathrm{~nm}$ laser. Lower panel insets are Raman spectra from strong and weak enhancement regions, circled in the Raman map. The maximum Raman intensity occurs for nanodisks in the 70 to $85 \mathrm{~nm}$ size range. (b) Similar SEM images (upper panel, the inset is from the boxed region) and Raman map (lower panel) of bow-tie arrays with sizes (triangle height) ranging from $20 \mathrm{to} 200 \mathrm{~nm}$ and inter-spacing (gap length) from 20 to $200 \mathrm{~nm}$. Bow-ties with 100-110 nm size emit the strongest Raman signals, again with less influence of bow-tie spacings in the current range.

For the nanodisks, the expected variation of the low loss energy spectrum with the electron probe position on/near the particles is confirmed (e.g., Fig. 2) [36]. At the disk edge and in an aloof position, the dipolar plasmon [42] is excited with an energy loss peak of $1.7 \mathrm{eV}$ for a $150 \mathrm{~nm}$ diameter disk on a $\mathrm{SiN}_{x}$ membrane, whereas at the center, the higher energy breathing mode $(2.3 \mathrm{eV})$ is predominant, which is not optically active [43]. The plasmon mode spatial distributions are also illustrated in Fig. 3b. It is the former which is known to be responsible for the Raman signal enhancement [44]. The peak positions are also in good agreement with those predicted theoretically using boundary element method analysis $[45,46]$.

Figure 3 compares the spectra and the spectrum images for several different sized disks with the same separation $(75 \mathrm{~nm})$ on a $S i N_{x}$ substrate. An increase (blue-shift) in dipolar plasmon energy with decreasing size is notable (as has been predicted theoretically [22]) in Fig. 3a. EELS maps of different sized nanodisks (Fig. 3b) at energy loss of 1.7, 1.9, and $2.1 \mathrm{eV}$ (using a $0.05 \mathrm{eV}$ energy window) are consistent with the corresponding energy loss peaks in Fig. 3. For instance, the maximum image intensity of the dipolar plasmon mode around the disk circumference for $90 \mathrm{~nm}$ disks occurs close to the EELS peak of $1.9 \mathrm{eV}$ (Fig. 3a). A complete set of spectrum image data in the range $1.0-2.3 \mathrm{eV}$ is given in the Supplementary Video 1, to which the reader is referred. It can be seen from these images that imperfections in the lithographic fabrication such as a protruding grain (Supplementary Fig. S5) can give rise to local higher plasmon intensities ("hot spots"). This indicates that non-smooth shapes might give rise to greater SERS enhancements.

There is much less variation of plasmon peak energy with varying spacing of same sized disks in the current range (Fig. 4). Thus, for $75 \mathrm{~nm}$ disks the peak energy varies only from $2.07 \mathrm{eV}$ (35 nm spacing) to $2.02 \mathrm{eV}$ (160 nm spacing). A more complete set of spectrum images is also shown in the Supplementary Video 2 . The slightly higher resonance energy for nanodisks with smaller inter-spacing is attributed to the favoring of the antibonding dipolar mode and repulsion of like charges [36, 47].

A comparison of the low loss EEL spectra around nanodisk perimeters with inter-spacing of $75 \mathrm{~nm}$ and sizes ranging from $50-190 \mathrm{~nm}$ for silica and silicon nitride substrates is shown in 


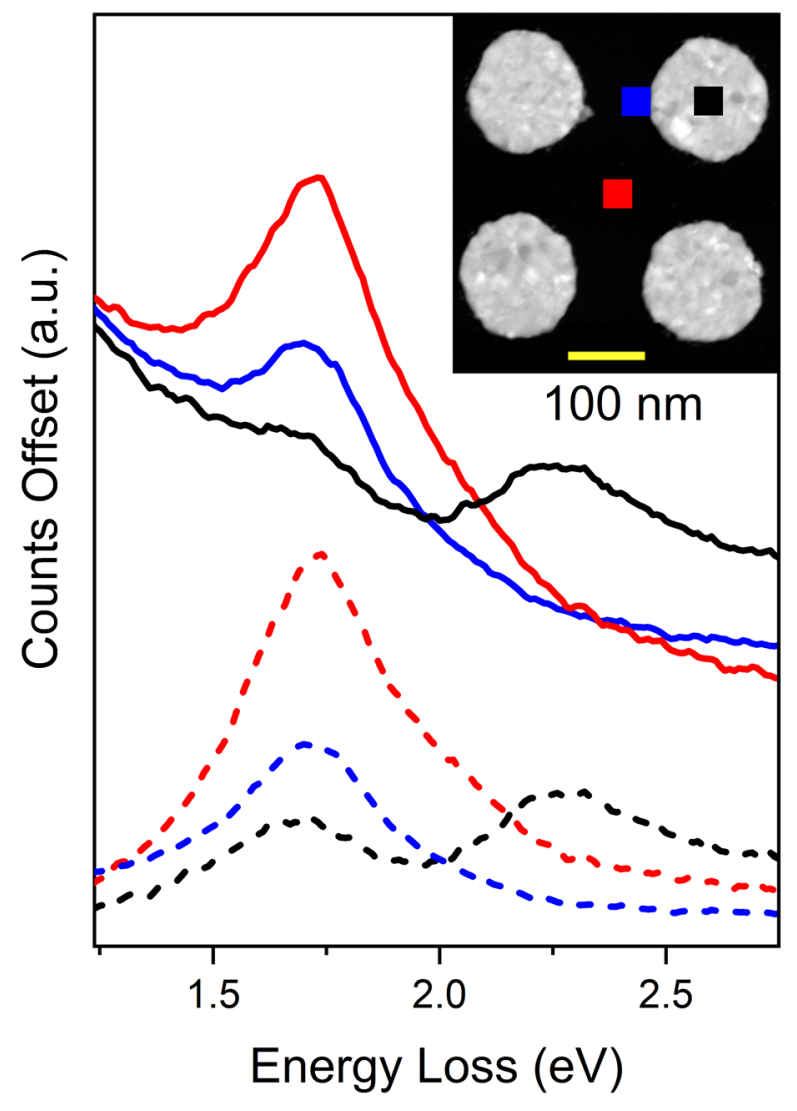

Figure 2: EEL spectra from a $150 \mathrm{~nm}$ diameter nanodisk array (separation $75 \mathrm{~nm}$ ) on a SiN $\mathrm{N}_{x}$ substrate at the disk center, edge, and the midpoint between the disks. Dashed lines are EELS after background subtraction. The inset shows a STEM annular dark field (ADF) image of the nanodisk array where the positions from which the corresponding EEL spectra were extracted are marked in the same color. The dipolar surface plasmon peak at $1.71 \mathrm{eV}$ is observed in all three locations, while the breathing (dark) mode is only observed at the disk center. Note that a polycrystalline microstructure of the gold nanoparticles is confirmed by the ADF contrast.

Fig. $5 a$ and $b$. The surface plasmon energies are plotted as a function of nanodisk size in Fig. 5c, along with those on Si substrates (Fig. 8a). It can be seen that the dipolar surface plasmon peak energies decrease linearly with increasing size and for the same size nanoparticles are higher for $\mathrm{SiO}_{x}$ which has a lower refractive index (1.5, 2 and 4 for $\mathrm{SiO}_{2}, \mathrm{Si}_{3} \mathrm{~N}_{4}$ and $\mathrm{Si}$ respectively) [30, 39]. For instance, for a plasmon peak energy of $1.9 \mathrm{eV}$, the disk diameter should be about $50 \mathrm{~nm}$ for silicon, $100 \mathrm{~nm}$ for silicon nitride, and about $150 \mathrm{~nm}$ for silica, and largely independent of inter-particle spacing.

The relationship between Raman intensity and surface plasmon energy/particle size as demonstrated in Fig. 1 can be investigated by varying the illuminating laser photon energy. Thus, with a $785 \mathrm{~nm}(1.58 \mathrm{eV})$ near-infrared laser, which is preferred medically, the Raman spectrum from R6G dye attached to 150 and $90 \mathrm{~nm}$ sized nanodisks on a $S_{i} N_{x}$ substrate separated $75 \mathrm{~nm}$ apart has relatively low intensity (Fig. 6a), consistent with the surface plasmon energies being $0.12 \mathrm{eV}$ and $0.32 \mathrm{eV}$ higher than the laser energy and so the surface plasmon is weakly excited. The Raman spectra of smaller nanoparticles with surface plasmon energies deviating further from the incident laser energy do not exhibit clear Raman signals other than noise, and therefore, they are not shown in Fig. 6a. For a $532 \mathrm{~nm}$ green laser (2.3 $\mathrm{eV}$ ), particles with surface plasmon peak energies closer to that of the illuminating photons give rise to higher intensity spectra (Fig. 6b) with a noticeable decline when the plasmon energy is much lower (e.g., for $150 \mathrm{~nm}$ diameter disks). For a $638 \mathrm{~nm}$ red laser $(1.9 \mathrm{eV})$, whose energy most closely matches that of the plasmon peak for $90 \mathrm{~nm}$ diameter disks, the markedly higher Raman signals for plasmon energies close to, or slightly lower than, the laser energy is clear (Fig. 7a). $150 \mathrm{~nm}$ nanodisks also have comparable Raman enhancement in the $1300-1600 \mathrm{~cm}^{-1}$ range, but have smaller intensities in the lower wavenumber range where the Raman scattered light energy deviates more (higher) from the surface plasmon energy. The Raman spectra of $75 \mathrm{~nm}$ nanodisks on $\mathrm{SiN}_{x}$ with different inter-spacing exhibit similar signal intensities (Fig. $7 \mathrm{~b}$ ) because their surface plasmon energies are similar to each other. Thus, the importance of matching the SERS illuminating laser energy to the nanoparticle plasmon peak energy is demonstrated and allows choice of the optimum laser energy-particle size combinations from this approach.

Finally, as the bulk data in Fig. 1 was generated for particles on a silicon substrate, a similar nanoparticle array was fabricated on a 15-nm-thick single crystalline silicon membrane. The energy loss and Raman spectra are shown in Fig. 8 for the 785 $\mathrm{nm}$ laser. The optimum combination is predicted to be a diameter and spacing of about $90 \mathrm{~nm}$ each, which is reasonably close to those shown visibly in Fig. 1. The surface plasmon energies of nanoparticles on crystalline $\mathrm{Si}$ membrane are systematically lower than those on $S_{i} N_{x}$ membranes (Figs. 3 and 4), following the same trend as observed earlier that higher refractive index of surrounding environment (4.0 versus 2.0 ) results in lower surface plasmon energy. Silicon, while being transparent at small thickness, is unlikely to be used as a nanoparticle coating, however, as it is less inert than silicon oxide or silicon nitride, although a recent study indicates low toxicity for silicon quantum dots [49]. Note that the bulk data are not normalized for the number of nanoparticles nor the area of the active plasmon regions, so an exact fit would not be expected.

\section{STEM-EELS and Raman data on bow-tie nanoparticles}

The current procedure was extended to more complex shapes such as bow-ties, for which local plasmon "hot spots" occur [50-52]. As indicated by the bulk data shown in Fig. 1, the bulk results are largely equivalent to those found for the nanodisks. 


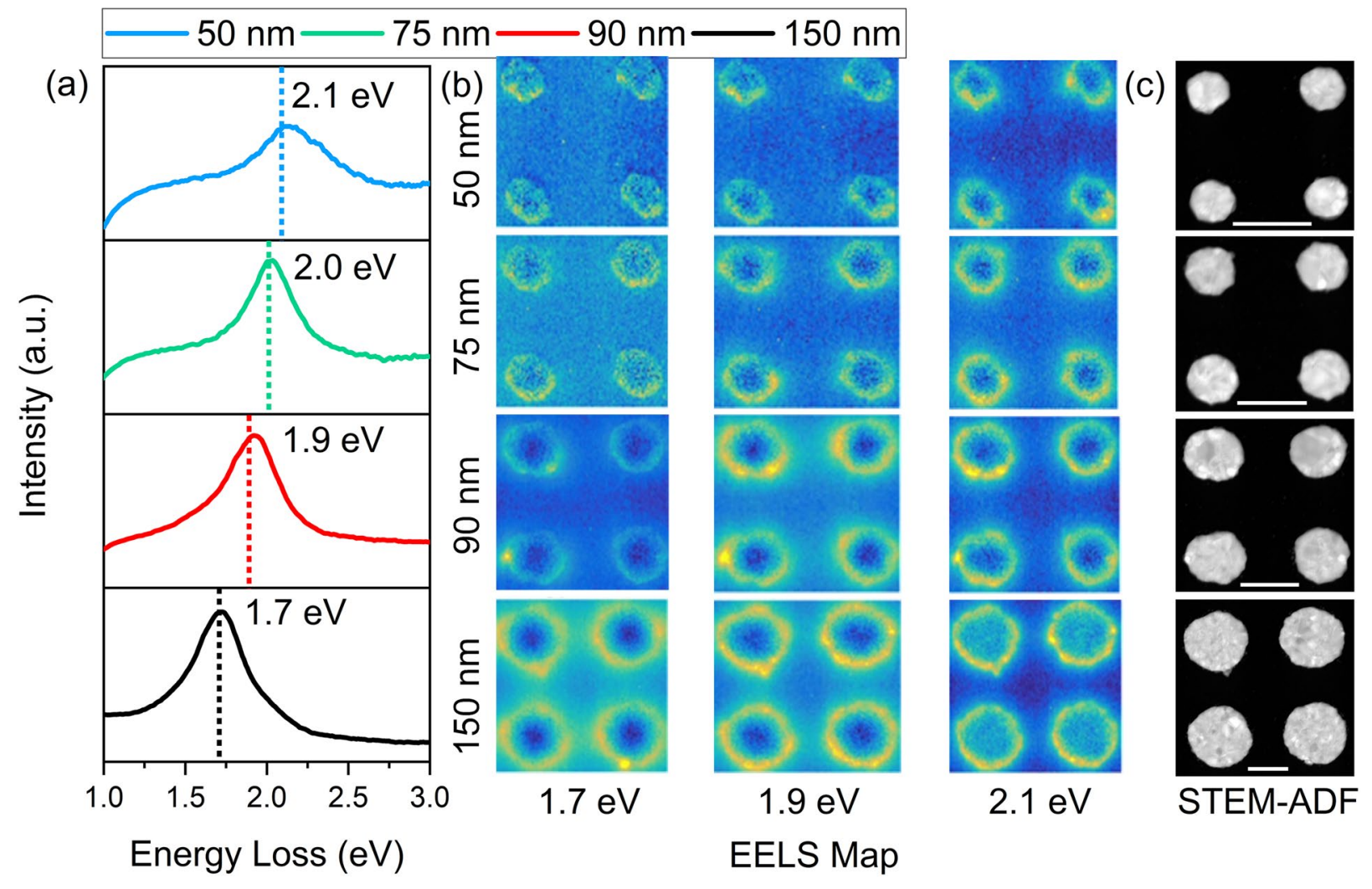

Figure 3: (a) Background subtracted EEL spectra at midpoints between disks and (b) the corresponding EELS maps at energy loss of 1.7, 1.9, and 2.1 $\mathrm{eV}$ (using a $0.05 \mathrm{eV}$ energy window) for $50,75,90$, and $150 \mathrm{~nm}$ sized nanodisk arrays on a $\operatorname{SiN}_{x}$ substrate (separation is constant at $75 \mathrm{~nm}$ ). An increase in the dipolar surface plasmon peak energy as the disks become smaller is notable in (a). This is further supported by the EELS maps in (b) where the edge of smaller disks show strong intensities at higher energy loss, closer to their plasmon peak energies. (c) STEM-ADF images of corresponding nanodisks in (b) showing four of the disks in the center of a $2 \times 2 \mu \mathrm{m}$ square region. Scale bars are $100 \mathrm{~nm}$.

The EELS data (Fig. 9) show two prominent plasmon peaks, one at the triangular prism corners and the other from the edge centers at higher energy, as reported previously $[36,32,53]$. The latter is not optically active [54], and so the prism corner energies are the most relevant here. Thus, for a $638 \mathrm{~nm}(1.94 \mathrm{eV})$ laser, the largest Raman signal of R6G is given for a nanoprism size of $140 \mathrm{~nm}$ (Fig. 9c) for which the laser energy is slightly higher than the plasmon energy. Inter-particle spacing plays a less significant role (Fig. 9d-f) (see Supplementary Fig. S3 for definitions of size and spacing for these nanoparticles). A direct comparison of the Raman spectra from bow-tie and disk nanoparticles for similar size (140 and $150 \mathrm{~nm}$, respectively) is shown in Fig. 10a, which indicates that the bow-ties have approximately two times higher intensity for the strongest peaks (e.g., 1360 $\mathrm{cm}^{-1}$ ), showing that nanoparticle shape is also an important parameter, as previously discussed $[21,23,55]$.

Using the electron beam lithography, the equilateral triangles in the bow-tie structures were replaced with isosceles triangles by changing the base length while maintaining a constant height of $140 \mathrm{~nm}$. The resulting Raman spectra on a bulk Si single crystalline substrate (Fig. 10b) show that smaller base length (i.e., sharper tips) results in stronger Raman signals due to the "lightning rod effect" [30], up to about $2 \times$ higher intensity for the sharpest ( $60 \mathrm{~nm}$ base) compared to right triangular bow-ties (280 $\mathrm{nm}$ base) at $1361 \mathrm{~cm}^{-1}$.

\section{Discussion}

By using electron beam lithographic fabrication techniques, we provide an experimental approach for systematically varying gold nanoparticle parameters to determine the most effective combination of particle and coating for optimizing surfaceenhanced Raman spectroscopy (SERS) signals. The data are correlated with the particle surface plasmon energies as determined by electron energy loss spectroscopy in a TEM on the exact same nanoparticles. Using this method any systematic errors from one batch to another are eliminated and direct spectral comparisons can be made. It is found that the maximum Raman intensity is obtained when the particle surface plasmon energy is close to, and slightly lower than, the energy of the illuminating laser energy stimulating the Raman signal. For instance, with a 785 $\mathrm{nm}$ laser $(1.6 \mathrm{eV})$ the optimum nanodisk diameter is estimated 


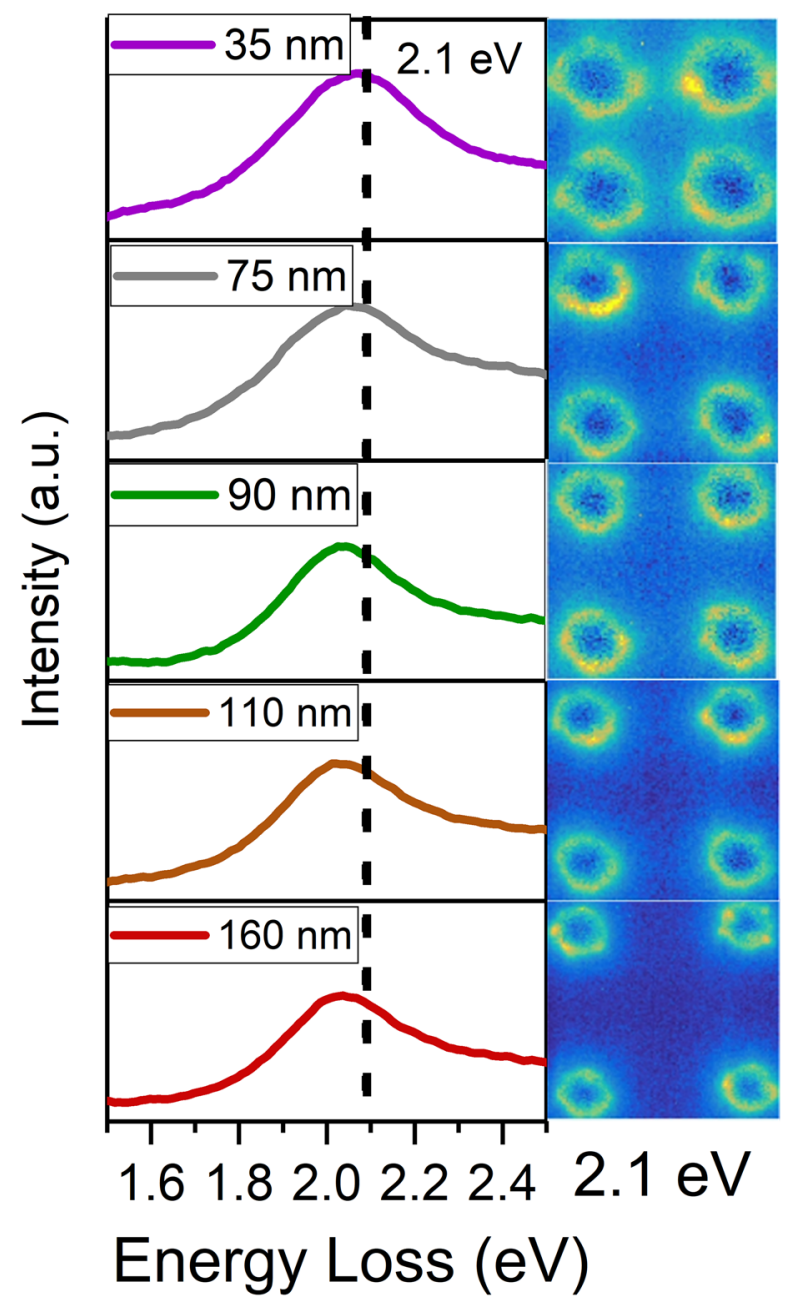

Figure 4: EEL spectra of $75 \mathrm{~nm}$ nanodisks with different inter-spacing extracted from the midpoint between nanodisks and the corresponding EELS maps at $2.1 \mathrm{eV}$. There is much less variation of plasmon peak energy with inter-spacing; therefore, the peripheries of the nanodisks show strong intensities at similar energy loss.

to be $190 \mathrm{~nm}$ for a $\mathrm{SiN}_{x}$ substrate, $220 \mathrm{~nm}$ for a $\mathrm{SiO}_{x}$ (based on Fig. 5b), and $90 \mathrm{~nm}$ for a silicon substrate. These values are reduced to 150 and $55 \mathrm{~nm}$ for the $\mathrm{SiO}_{x}$ substrate for 638 (1.94 $\mathrm{eV})$ and $532 \mathrm{~nm}(2.33 \mathrm{eV})$ laser wavelengths respectively, consistent with smaller nanoparticles and larger refractive index substrates having higher surface plasmon energies, as might be expected $[30,39]$. It can be noted that lower wavelength lasers (higher energy) allow smaller nanoparticles to achieve this resonance effect, which may have other advantages as well, such as easier transportation to tumor regions and higher cellular uptake [56].

We have also begun to explore the influence of nanoparticle shape. Thus bow-tie structures have up to $100 \%$ higher Raman signal for the signature $1361 \mathrm{~cm}^{-1}$ peak of R6G dye compared to the more simple nanodisks of the same equivalent size, most likely due to the angular features and possible hot spots [54]. By decreasing one apex angle (i.e., the particle shape is "sharper"), the Raman signal is further enhanced by about a factor of 2 . This has also been found in altering spherical gold particles to nanorod shapes where up to a ten-fold improvement in SERS signal was reported [21]. It is clear that manipulation of the particles into more complex shapes (e.g., nanostars [57]) is warranted to explore further optimization of the Raman signal.

The current spherical gold-silica nanoparticles clearly work well and readily pass along vascular passages in the blood stream e.g., [28]. It is unlikely that this would be duplicated by nanoparticles which replicate the shape of their inner gold core (e.g., nanodisks). However by suitable preparation the overall spherical shape can be maintained with the protective coating providing the composite nanospheres. An optically transparent material for the chosen laser wavelength is preferable, and so silicon nitride is superior to silica for smaller gold nanoparticles (and hence smaller possible overall size).

The design then of the most effective nanoparticles for SERS involves the combination of a number of factors. The approach taken here is to provide a scientific underpinning to focus on optimization of nanoparticle parameters, coating material, illuminating laser etc. rather than using a trial-and-error multiplex approach involving a large number of physical permutations. The correlation of SERS intensity with plasmon energies as determined by electron energy loss spectroscopy in a STEM is clearly established, and the efficacy of electron beam lithography to provide reproducible nanoparticle arrays and shapes is demonstrated. Once the best combination is chosen, scaling up to fabricate useful quantities of nanoparticles (e.g., by photolithography, imprinting or chemical synthesis) will be the next challenge.

It is also interesting to suggest some medical problems whereby the current approach might be used to best effect (see Table 1). One of demonstrated capabilities of SERS is to detect colon cancer using an endoscopic system [19, 20]. Optimizing SERS nanoparticles and their complementing illumination laser energies to enhance the Raman signals would contribute significantly to the detection sensitivity of the endoscopy. SERS nanoparticles can also be implemented as imaging agents to delineate tumors during surgical operations, signaling cancerous regions that are difficult to see (e.g., brain tumors [17]). Recently, plasmonic Au nanoparticles have also been used in photothermal therapy for treatment of brain tumors (glioblastoma) and light activated drug delivery $[58,59]$. Resonating these nanoparticles with near-infrared (NIR) radiation is the key to deliver maximum heat to tumor regions. Whether or not the nanoparticles are bio-functionalized on the outer surface is unlikely to affect the surface plasmon resonances as the outer environment is separated from the active gold by the inorganic coating layer (which needs to be verified experimentally). 

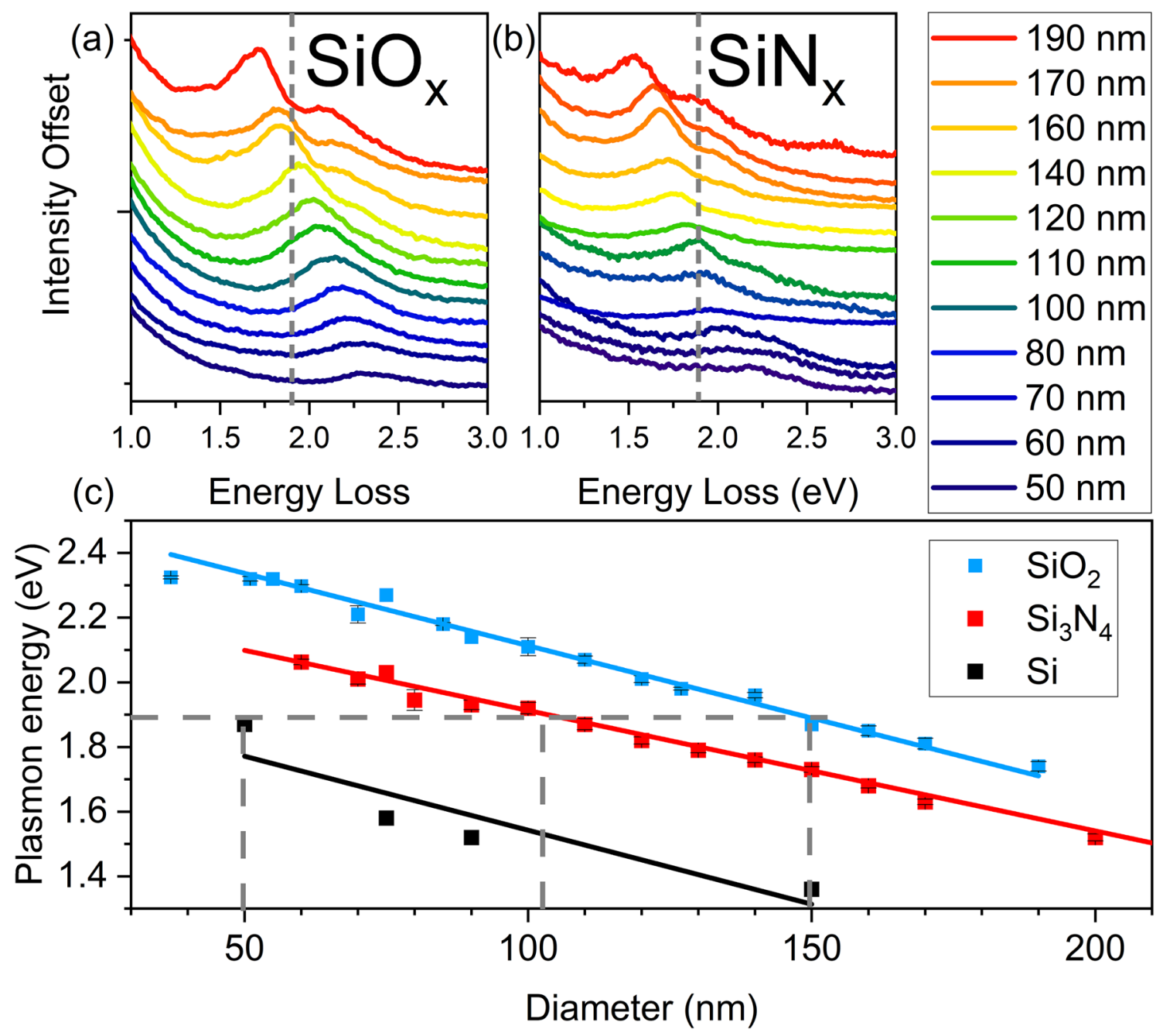

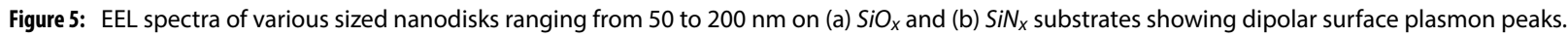
Higher order plasmon modes (additional peaks at higher energy loss) are also observed for large nanodisks with diameters greater than $150 \mathrm{~nm}$. EELS were extracted around the disk perimeters. The dipolar surface plasmon peak energy of the same size nanodisks is measured to be lower for $\operatorname{SiN}_{x}$ which has a higher refractive index. The plasmon peak positions in (a) and (b) are used to compose (c) showing the surface plasmon energy as a function of nanodisk size on $\mathrm{SiN}_{x}$ and $\mathrm{SiO}_{x}$ substrates. The data for a silicon substrate are also plotted based on Fig. 8a. This plot can be used to predict optimum nanoparticle sizes for specific plasmon peak energies, as indicated for the $1.9 \mathrm{eV}$ value. The surface plasmon energy varies approximately linearly with nanodisk size for the range from 50 to $200 \mathrm{~nm}$, but is upper bound by the Fröhlich condition for smaller nanoparticles [48]. The surface plasmon energy decreases for the same sized nanodisks on $\mathrm{SiO}_{x}, \mathrm{SiN}_{x}$, and $\mathrm{Si}$ substrates, respectively.

In addition to cancer detection, SERS based immunoassays are compatible with a wide range of biomarkers, such as cardiac troponin I and myoglobin for myocardial infraction diagnosis [60] and bisphenol A (BPA) for blood toxins detection [61]. There are many other possibilities that can be speculated upon.

\section{Conclusions}

1. Electron beam lithography provides a straightforward means of systematically varying nanoparticle parameters such as size, shape, separation etc. to create arrays of gold nanoparticles in order to test their effectiveness for produc- ing surface-enhanced Raman spectroscopy (SERS) signals for possible medical applications.

2. The surface plasmon energies which induce the SERS effect are established on the same nanoparticles using electron energy loss spectroscopy in an scanning transmission electron microscope (STEM-EELS).

3. The largest Raman signal intensities are obtained when the illuminating laser energy coincides with, or is slightly higher than, the Au nanoparticle surface plasmon energies, with size and shape having the most important effects.

4. The influence of various dielectric substrates is established, with higher refractive index allowing smaller nanoparticle sizes to yield the highest Raman signals. 

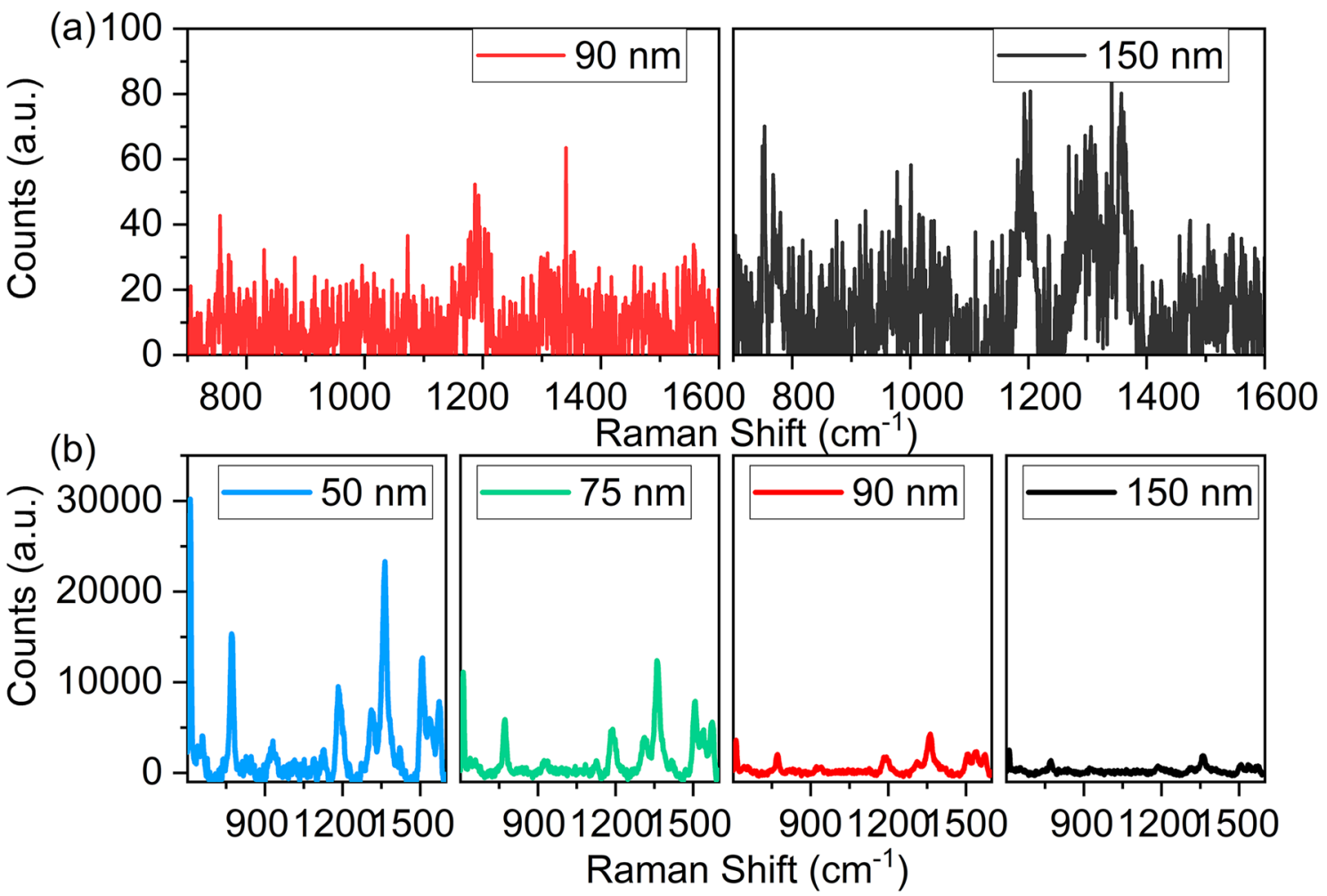

Figure 6: Raman spectra of R6G attached to nanodisks of different size (at a constant separation of $75 \mathrm{~nm}$ on a SiN $\mathrm{N}_{x}$ substrate) measured using (a) a 785 $\mathrm{nm}$ near-infrared laser and (b) a $532 \mathrm{~nm}$ green laser. Raman spectra are on the same scale as the left most spectrum. Spectra in (a) are noisy because the incident laser energy (1.58 eV) is lower than the surface plasmon energies (Fig. 3a), resulting in weak excitation of surface plasmons. Spectra of $55 \mathrm{~nm}$ disks in (b) exhibit the strongest Raman signal owing to the closer match between the surface plasmon energy of 2.1 eV (Fig. $3 a$ ) and the illuminating laser energy $(2.3 \mathrm{eV})$.

5. For gold nanodisks, the optimum diameters for highest Raman signals in this study, based on the plasmon peak energies determined by STEM-EELS, are predicted to be approximately 60,150 , and $230 \mathrm{~nm}$ for 532, 638, and 785 $\mathrm{nm}$ wavelength lasers, respectively, $(2.3,1.9,1.6 \mathrm{eV}$ energies) on a silicon oxide substrate; 100 and $200 \mathrm{~nm}$ for 638 and $785 \mathrm{~nm}$ wavelength lasers on a silicon nitride substrate; and 50 and $90 \mathrm{~nm}$ for 638 and $785 \mathrm{~nm}$ wavelength lasers on a crystalline silicon substrate.

6. The Raman signal intensity is increased by approximately a factor of 2 using bow-tie nanoparticles compared to nanodisks and about a further factor of 2 by fabricating "sharper" triangular nanoparticles.

7. The current approach can be employed to design nanoparticle combinations to generate the largest physical signals such as SERS for the detection of medical-related issues such as cancerous tumors and circulating tumor cells/DNA, cardiac troponin I, and bisphenol A in blood toxins.

\section{Experimental details}

\section{Nanoparticle fabrication}

$2 \times 2 \mathrm{~cm}$ chips cut from a pristine single crystal (100) silicon wafer $\left(\mathrm{VWR}^{T M}\right.$ ) (no particular surface treatment other than cleaning, expected native oxide thickness is approximately 2 $\mathrm{nm}$ ) were coated with approximately $70 \mu \mathrm{m}$ of PMMA (polymethyl methacrylate) and patterned in a JBX-6300FS electron beam (e-beam) lithography system (JEOL Ltd., Japan) operating at $100 \mathrm{kV}$ and $500 \mathrm{pA}$ beam current. An overall writing precision of approximately $10 \mathrm{~nm}$ was typically achieved based on the manufacturer's specifications. The array was designed using KLayout and fractured using BEAMER (GenISys GmbH, Germany) software. 30-nm-thick gold thin films were deposited using a KJL e-beam evaporator (Kurt J. Lesker Company, USA) onto the patterned substrate with an evaporated organic adhesion layer of (3-mercaptopropyl) trimethoxysilane, which has minimal plasmon damping effects compared with metallic 

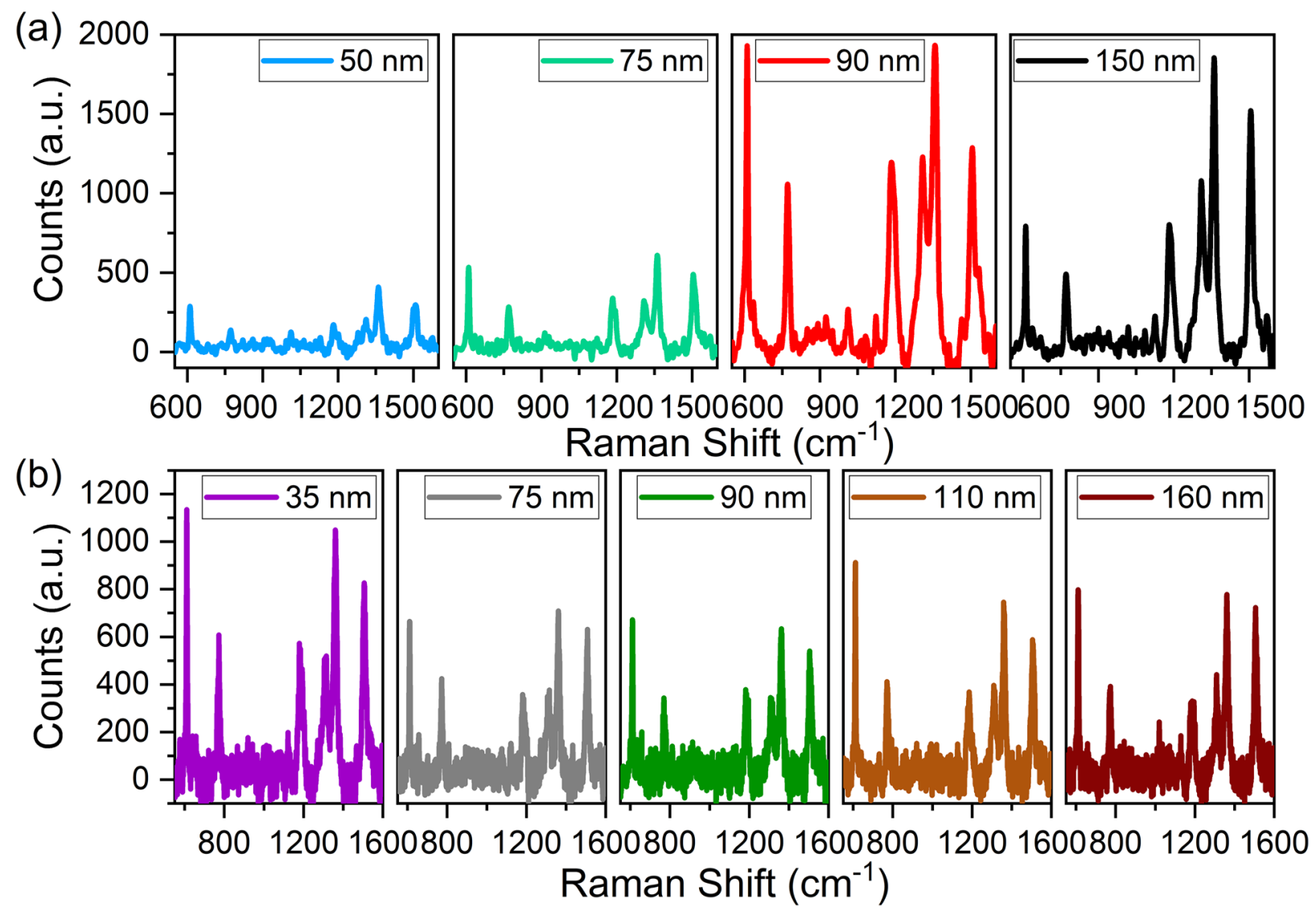

Figure 7: Raman spectra of R6G attached to nanodisk arrays on a $\operatorname{SiN}_{x}$ substrate with different (a) size and (b) inter-spacing measured using a 638 nm laser. $90 \mathrm{~nm}$ sized disks have overall the strongest Raman enhancement, while inter-spacing has a lesser effect on the enhancement. Raman spectra are on the same scale as the left most spectrum.

adhesion layers such as $\mathrm{Ti}$ and $\mathrm{Cr}$, as previously reported in [38]. The underlying PMMA was removed by acetone, leaving the nanoparticles on the substrate.

Supplementary Fig. S2 shows the pattern design and SEM pictures of the resultant arrays. The parameters defining the nanodisk and bow-tie arrays are shown in Supplementary Fig. S3.

A similar procedure was carried out for arrays fabricated onto TEM grids coated with silica, silicon nitride, and single crystalline (100) silicon thin films (Ted Pella Inc., USA). The same underlying adhesive layer of (3-mercaptopropyl) trimethoxysilane is used. The $15-\mathrm{nm}$-thick membranes are supported by a $0.5 \times 0.5 \mathrm{~mm}$ meshed window with an aperture size of $50 \times 50$ $\mu \mathrm{m}$. Note that the surface of single crystalline Si membrane TEM grids is hydrophobic, causing trouble with the e-beam resist (PMMA) deposition; therefore, grids were glow discharged for 30 seconds in oxygen/argon plasma before fabrication. Because of the delicate nature of these films, the fabrication was more difficult to perform, but particular attention to grid handling and feature aligning allowed the fabrication of $30 \times 40 \mu \mathrm{m}$ areas with $2 \times 2 \mu \mathrm{m}$ nanoparticle arrays. This approach allows the direct comparison of nanoparticles made in the same batch and avoids any artifacts which might be created, for instance in focused ion beam-fabricated samples. Supplementary Fig. S4 shows a typical array layout for a silicon oxide membrane substrate.

\section{Raman spectroscopy}

Samples were incubated with $0.1 \mathrm{mg} / \mathrm{mL}$ Raman dye (thiophenol) de-ionized (DI) water solution overnight and subsequently rinsed with DI water prior to measurements. Raman spectroscopy maps attached to bulk samples were acquired using a Horiba LabRAM HR Evolution confocal Raman microscope through an automated stage. The stepping size of the stage is 2 $\mu \mathrm{m}$ and the laser is a $785 \mathrm{~nm}$ infrared laser with an output power of approximately $20 \mathrm{~mW}$ and exposure time of $5 \mathrm{~s}$. 
Raman Shift (eV)

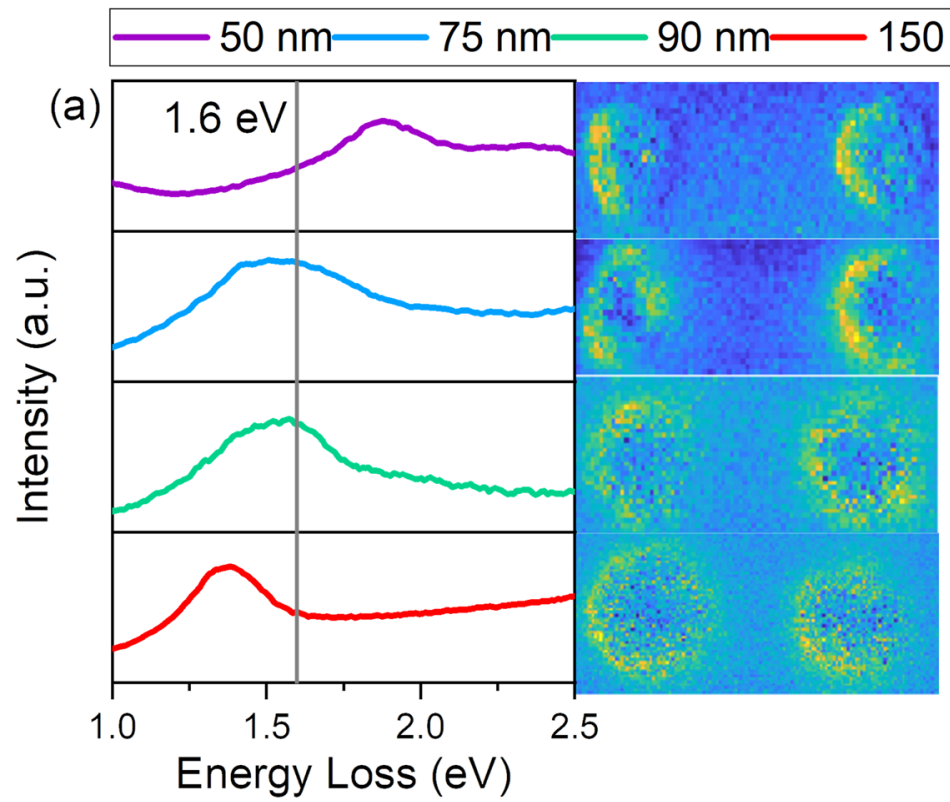

$\begin{array}{llllll}0.07 & 0.10 & 0.12 & 0.15 & 0.17 & 0.20\end{array}$

ביב

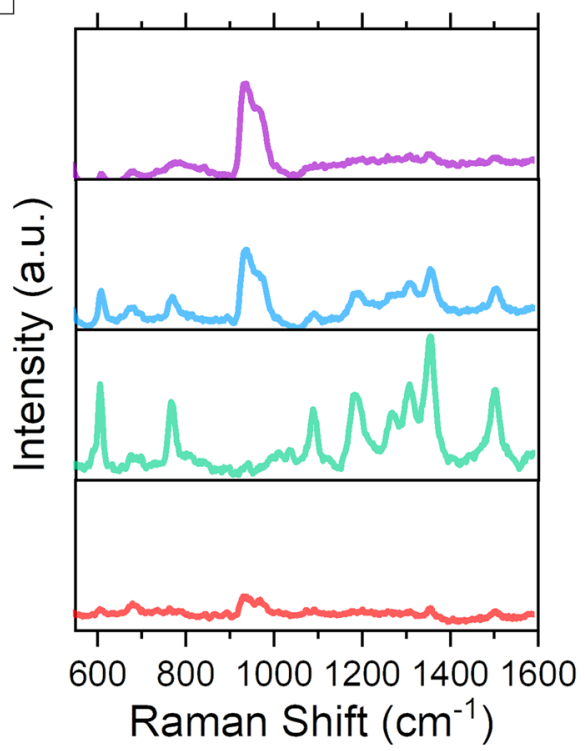

(b)
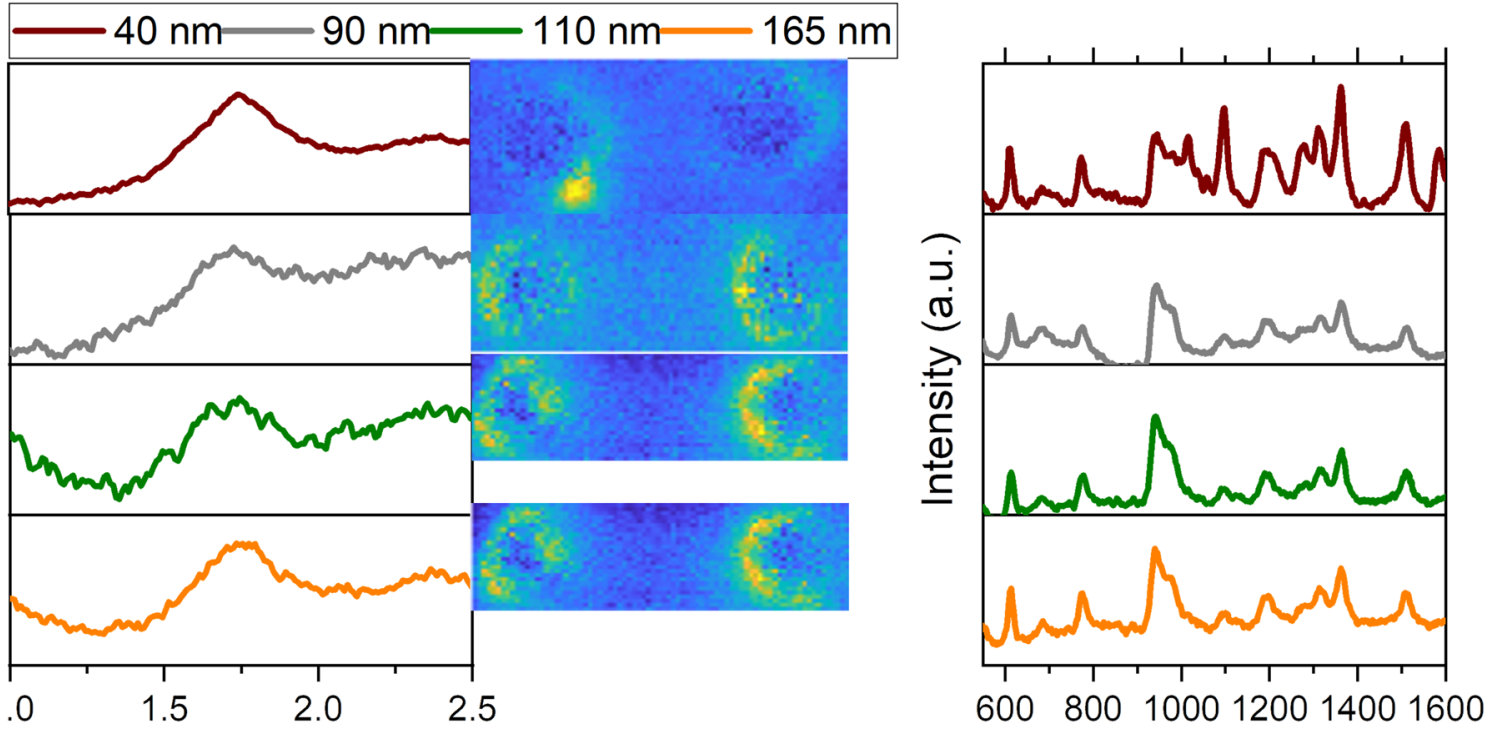

Energy Loss $(\mathrm{eV})$

Raman Shift $\left(\mathrm{cm}^{-1}\right)$

Figure 8: (a) EEL spectra (left panel), EELS maps at $1.6 \mathrm{eV}$ (middle panel) and the corresponding Raman spectra (right panel) of 50, 75, $90 \mathrm{and} 150 \mathrm{~nm}$ nanodisks on a single crystalline $\mathrm{Si}(100)$ thin film substrate. The EELS are extracted from the midpoint between nanodisks, and the Raman spectra are measured using a $785 \mathrm{~nm}(1.58 \mathrm{eV})$ infrared laser. Strongest Raman signal enhancement is achieved with $90 \mathrm{~nm}$ diameter nanodisks, which have a dipolar plasmon energy of $1.6 \mathrm{eV}$, matching that of the laser excitation energy. The Raman peak from 900 to $1000 \mathrm{~cm}^{-1}$ is from the substrate, which is stronger for smaller sized nanodisk arrays (upper figures) because more substrate area is illuminated. (b) EEL spectra (left panel), EELS maps at $1.7 \mathrm{eV}$ (middle panel) and the corresponding Raman spectra (right panel) of $75 \mathrm{~nm}$ diameter nanodisks with different separation distance on a Si substrate. Inter-spacing has only a minor effect on surface plasmon energy, but overall Raman enhancement is observed to be stronger for smaller inter-spacing with more nanoparticles in the irradiated area.

For the TEM samples, Raman spectra acquisition was carried out after the TEM analysis so that electron beam decomposition of the organic dye did not influence either the TEM or Raman data. The same arrays from which EELS had been collected were located by correlating grid position and measured manually on the Raman system using 785, 638, and $532 \mathrm{~nm}$ lasers, with a spot size of $1 \mu \mathrm{m}$ as the TEM samples are much smaller and, therefore, need precise maneuvering. Since the TEM grid is optically transparent, a much more sensitive Raman dye rhodamine $6 \mathrm{G}(0.1 \mathrm{mg} / \mathrm{mL})$ is used to produce the 


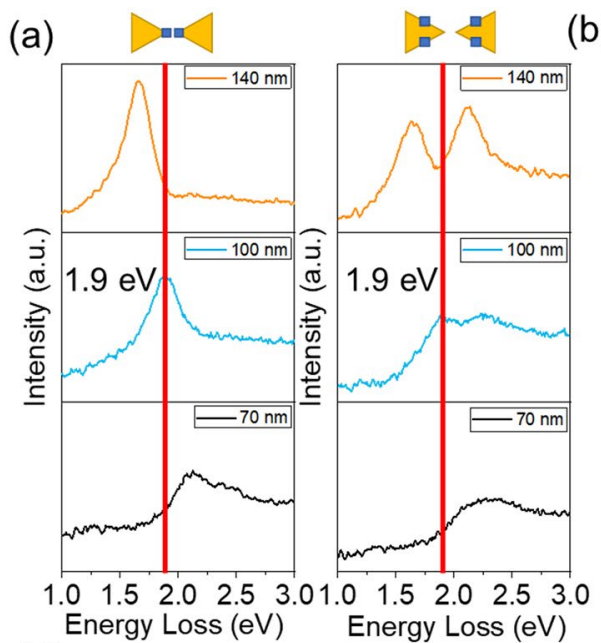

(d)

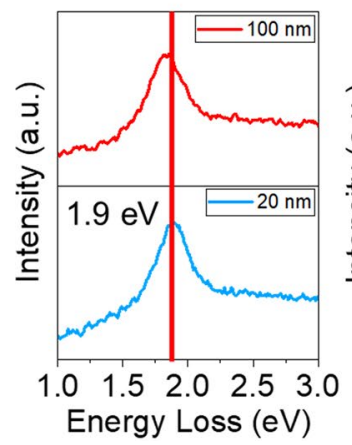

(b) $1.65 \mathrm{eV}$

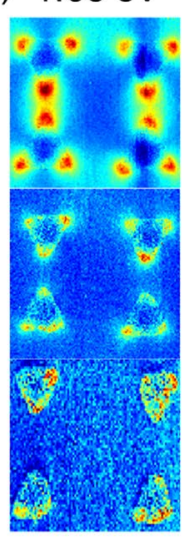

(e)

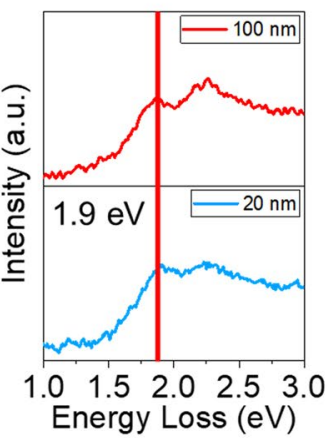

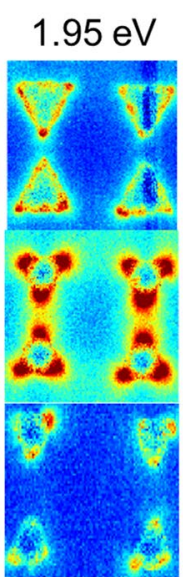

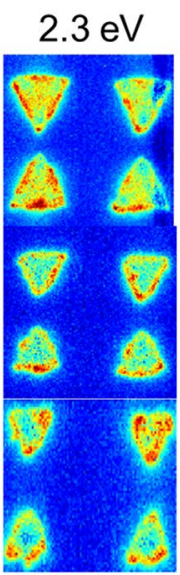

(c)

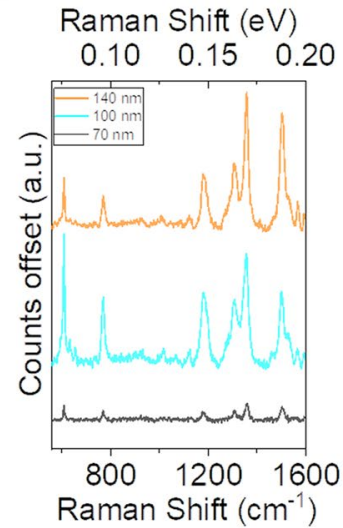

Figure 9: (a) EEL spectra of bow-tie arrays on a $\operatorname{SiN}_{x}$ substrate with the triangle height of 70, 100, and $140 \mathrm{~nm}$ extracted from the tip region (left) showing dipolar surface plasmon peaks and the edge region (right) showing additional quadrupolar surface plasmon peaks at higher energies. (b) EELS maps of the corresponding bow-tie arrays in (a) at energy loss of 1.65, 1.95, and $2.3 \mathrm{eV}$. The dipolar surface plasmon peak is observed mainly at bow-tie vertices, with higher peak energy for smaller triangle height. The quadrupolar peak is only observed at the triangle edge and is not optically active [36]. (c) The corresponding Raman spectra of the bow-tie arrays in (a) measured using a $638 \mathrm{~nm}$ (1.9 eV energy) laser. 140-nm-high bow-ties have strongest Raman enhancement at $1360 \mathrm{~cm}^{-1}$. (d) EEL spectra of bow-ties at a constant height of $100 \mathrm{~nm}$, separated 20 and $100 \mathrm{~nm}$ apart on a SiN ${ }_{x}$ substrate, with the corresponding EELS maps shown in (e) and Raman spectra measured using a $638 \mathrm{~nm}$ ( $1.9 \mathrm{eV}$ energy) laser shown in (f). Smaller gap distance results in a slight blue-shift of surface plasmon energy, but overall shows similar Raman enhancement.

Raman signal. Lower output power for higher energy lasers $(0.6 \mathrm{~mW}, 1.7 \mathrm{~mW}, 8 \mathrm{~mW}$ for $532 \mathrm{~nm}, 638 \mathrm{~nm}$ and $785 \mathrm{~nm}$ lasers respectively) is used to avoid ablation effects [46].

\section{Scanning transmission electron microscopy-electron energy loss spectroscopy (STEM-EELS)}

The STEM images and electron energy loss spectra were obtained in a monochromated, image-corrected (but not probe corrected) Titan environmental TEM (Thermo Fisher Scientific) operated at $300 \mathrm{kV}$ with a convergence semi-angle of $8.4 \mathrm{mrad}$ and an estimated probe size of $0.5 \mathrm{~nm}$ in diameter. The EEL spectrometer was a GIF Quantum (Gatan Inc.) with a collection angle of $18.3 \mathrm{mrad}$. EEL spectrum images (100 $\times 100$ pixels) were collected with a pixel size of $2-4 \mathrm{~nm}$ (we used smaller pixel size for smaller structures to keep acquisition time fairly constant), a dispersion of $0.01 \mathrm{eV} / \mathrm{channel}$, and a dwell time of 2 ms per pixel. The full width half maxima (FWHM) of the zero loss peak (ZLP) across the whole spectrum images is less than $0.2 \mathrm{eV}$. The ZLP was subtracted using the fitted logarithm tail method implemented in DigitalMicrograph (Gatan Inc.). EEL spectra were integrated from regions of interest after ZLP subtraction. Selected area dffraction patterns (SADP) in TEM mode confirm that the gold is polycrystalline.

\section{Acknowledgments}

This work was supported by the Center for Cancer Nanotechnology Excellence for Translational Diagnostics (CCNETD) at Stanford University through an award (Grant No. U54 CA199075) from the National Cancer Institute (NCI) of the National Institutes of Health (NIH). We also acknowledge 

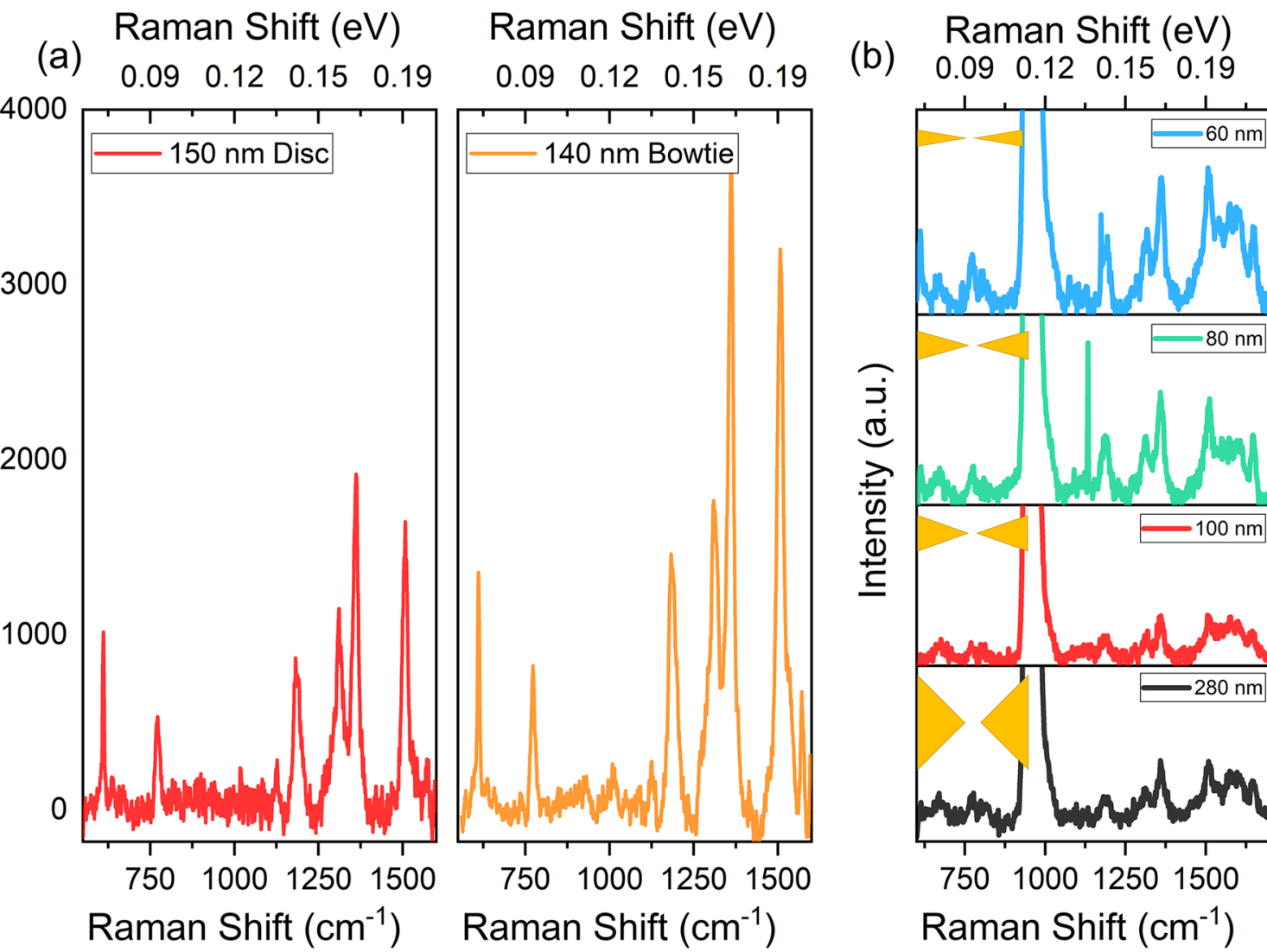

Figure 10: (a) A direct comparison of the Raman spectra of R6G attached to $150 \mathrm{~nm}$ diameter nanodisks (left) and $140 \mathrm{~nm}$ sized bow-ties (right) on $\operatorname{SiN}_{x}$ substrate measured using a $638 \mathrm{~nm}$ laser. The vertical axes have the same scale. The bow-tie structure exhibits clearly higher intensity Raman signals, up to $100 \%$ for the $1360 \mathrm{~cm}^{-1}$ Raman peak. (b) Raman spectra of bow-tie arrays comprised of isosceles triangles with base length of 60,80 , 100 , and $280 \mathrm{~nm}$ and a constant height of $140 \mathrm{~nm}$ (schematics inset on the left). The structures were fabricated on a bulk crystalline silicon (100) wafer substrate, and a $785 \mathrm{~nm}$ laser is used for illumination. Arrays with smaller base length (i.e., sharper tips) exhibit stronger Raman signals. The "off-scale" peaks are from the silicon substrate.

TABLE 1: A summary of possible medical applications of SERS active gold nanoparticles.

\begin{tabular}{|c|c|c|c|}
\hline Modality & Application & Functionalization & References \\
\hline Detection & $\begin{array}{l}\text { Endoscopy for colon } \\
\text { cancer }\end{array}$ & $\mathrm{N} / \mathrm{A}$ & {$[19,20]$} \\
\hline Imaging & Brain tumors & Maleimide-DOTA-Gd & [17] \\
\hline Treatment & $\begin{array}{l}\text { Wireless photothermal } \\
\text { treatment for glioblas- } \\
\text { toma }\end{array}$ & $\mathrm{N} / \mathrm{A}$ & [58] \\
\hline Treatment & $\begin{array}{l}\text { Light activated drug } \\
\text { delivery }\end{array}$ & Mesoporous silica & [59] \\
\hline Detection & Myocardial infraction & Cardiac Troponin I & {$[60]$} \\
\hline Detection & Blood toxins & Bisphenol A & {$[61]$} \\
\hline
\end{tabular}

the use of the Stanford Nano Shared Facility (SNSF), partially supported by the National Science Foundation under award ECCS-1542152. We (RS, YZ, SJM) would like to acknowledge the inspiring ideas and contributions of our collaborator (SSG) to the approach and experiments described herein. Dr. Gambhir sadly passed away during completion of this manuscript. We appreciate, in addition, the helpful discussions with Dr. Hamed Arami and Professor Utkan Demirci.

\section{Data Availability}

The datasets generated during and/or analysed during the current study are availablefrom the corresponding author on reasonable request.

\section{Declarations}

Conflict of interest On behalf of all authors, the corresponding author states that there is no conflict of interest.

\section{Supplementary Information}

The online version contains supplementary material available athttps://doi.org/10.1557/s43578-021-00320-4. 


\section{References}

1. A.B. Chinen, C.M. Guan, J.R. Ferrer, S.N. Barnaby, T.J. Merkel, C.A. Mirkin, Nanoparticle probes for the detection of cancer biomarkers, cells, and tissues by fluorescence. Chem. Rev. 115(19), 10530-10574 (2015). https://doi.org/10.1021/acs. chemrev.5b00321

2. J.D. Heidel, M.E. Davis, Clinical developments in nanotechnology for cancer therapy. Pharm. Res. 28(2), 187-199 (2011). https://doi.org/10.1007/s11095-010-0178-7

3. J.V. Jokerst, T. Lobovkina, R.N. Zare, S.S. Gambhir, Nanoparticle PEGylation for imaging and therapy. Nanomedicine 6(4), 715-728 (2011). https://doi.org/10.2217/nnm.11.19

4. J.L. Perry, K.P. Herlihy, M.E. Napier, J.M. DeSimone, PRINT: A novel platform toward shape and size specific nanoparticle theranostics. Acc. Chem. Res. 44(10), 990-998 (2011). https:// doi.org/10.1021/ar2000315

5. R.A. Petros, J.M. DeSimone, Strategies in the design of nanoparticles for therapeutic applications. Nat. Rev. Drug Discov. 9(8), 615-627 (2010). https://doi.org/10.1038/nrd2591

6. B.R. Smith, S.S. Gambhir, Nanomaterials for in vivo imaging. Chem. Rev. 117(3), 901-986 (2017). https://doi.org/10.1021/ acs.chemrev.6b00073

7. A. de la Zerda, J.W. Kim, E.I. Galanzha, S.S. Gambhir, V.P. Zharov, Advanced contrast nanoagents for photoacoustic molecular imaging, cytometry, blood test and photothermal theranostics. Contrast Media Mol. Imaging 6(5), 346-369 (2011). https://doi.org/10.1002/cmmi.455

8. C.M. Earhart, C.E. Hughes, R.S. Gaster, C.C. Ooi, R.J. Wilson, L.Y. Zhou, E.W. Humke, L. Xu, D.J. Wong, S.B. Willingham, E.J. Schwartz, I.L. Weissman, S.S. Jeffrey, J.W. Neal, R. Rohatgi, H.A. Wakelee, S.X. Wang, Isolation and mutational analysis of circulating tumor cells from lung cancer patients with magnetic sifters and biochips. Lab Chip 14(1), 78-88 (2014). https://doi.org/10.1039/c3lc50580d

9. M.L. Schipper, G. Iyer, A.L. Koh, Z. Cheng, Y. Ebenstein, A. Aharoni, S. Keren, L.A. Bentolila, J. Li, J. Rao, X. Chen, U. Banin, A.M. Wu, R. Sinclair, S. Weiss, S.S. Gambhir, Particle size, surface coating, and PEGylation influence the biodistribution of quantum dots in living mice. Small 5(1), 126-134 (2009). https://doi.org/10.1002/smll.200800003

10. M. Fleischmann, P.J. Hendra, A.J. McQuillan, Raman spectra of pyridine adsorbed at a silver electrode. Chem. Phys. Lett. 26(2), 163-166 (1974)

11. K. Kneipp, H. Kneipp, I. Itzkan, R.D. Ramachandra, S.F. Michael, Surface-enhanced Raman scattering and biophysics. J. Phys. 597(18), 597-624 (2002)

12. K. Kneipp, Y. Wang, H. Kneipp, L.T. Perelman, I. Itzkan, R.R. Dasari, M.S. Feld, Single molecule detection using surface-enhanced Raman scattering (SERS). Phys. Rev. Lett. 78(9), 1667 (1997)
13. S. Nie, S.R. Emory, Probing single molecules and single nanoparticles by surface-enhanced Raman scattering. Science 275(5303), 1102-1106 (1997). https://doi.org/10.1126/science.275.5303. 1102

14. Z.A. Nima, M. Mahmood, Y. Xu, T. Mustafa, F. Watanabe, D.A. Nedosekin, M.A. Juratli, T. Fahmi, E.I. Galanzha, J.P. Nolan, A.G. Basnakian, V.P. Zharov, A.S. Biris, Circulating tumor cell identification by functionalized silver-gold nanorods with multicolor, super-enhanced SERS and photothermal resonances. Sci. Rep. 4, 1-8 (2014). https://doi.org/10.1038/srep04752

15. B. Sharma, R.R. Frontiera, A.I. Henry, E. Ringe, R.P. Van Duyne, SERS: Materials, applications, and the future. Mater. Today 15(1-2), 16-25 (2012)

16. H. Arami, C.B. Patel, S.J. Madsen, P.J. Dickinson, R.M. Davis, Y. Zeng, B.K. Sturges, K.D. Woolard, F.G. Habte, D. Akin, R. Sinclair, S.S. Gambhir, Nanomedicine for spontaneous brain tumors: a companion clinical trial. ACS Nano 13(3), 2858-2869 (2019). https://doi.org/10.1021/acsnano.8b04406

17. M.F. Kircher, A. De La Zerda, J.V. Jokerst, C.L. Zavaleta, P.J. Kempen, E. Mittra, K. Pitter, R. Huang, C. Campos, F. Habte, R. Sinclair, C.W. Brennan, I.K. Mellinghoff, E.C. Holland, S.S. Gambhir, A brain tumor molecular imaging strategy using a new triple-modality MRI-photoacoustic-Raman nanoparticle. Nat. Med. 18(5), 829-834 (2012). https://doi.org/10.1038/nm.2721

18. C.L. Zavaleta, B.R. Smith, I. Walton, W. Doering, G. Davis, B. Shojaei, M.J. Natan, S.S. Gambhir, Multiplexed imaging of surface enhanced Raman scattering nanotags in living mice using noninvasive Raman spectroscopy. Proc. Natl. Acad. Sci. 106(32), 13511-13516 (2009). https://doi.org/10.1073/pnas.0813327106

19. C.L. Zavaleta, K.B. Hartman, Z. Miao, M.L. James, P.J. Kempen, A.S. Thakor, C.H. Nielsen, R. Sinclair, Z. Cheng, S.S. Gambhir, Preclinical evaluation of Raman nanoparticle biodistribution for their potential use in clinical endoscopy imaging. Small 7(15), 2232-2240 (2011). https://doi.org/10.1002/smll.201002317

20. C.L. Zavaleta, M.F. Kircher, S.S. Gambhir, Raman's 'effect' on molecular imaging. J. Nucl. Med. 52(12), 1839-1844 (2011)

21. J.V. Jokerst, A.J. Cole, D. Van de Sompel, S.S. Gambhir, Gold nanorods for ovarian cancer detection with photoacoustic imaging and resection guidance via Raman imaging in living mice. ACS Nano 6(11), 10366-10377 (2012)

22. K.L. Kelly, E. Coronado, L.L. Zhao, G.C. Schatz, The optical properties of metal nanoparticles: the influence of size, shape, and dielectric environment. J. Phys. Chem. B 107(3), 668-677 (2003). https://doi.org/10.1021/jp026731y

23. B.R. Smith, P.J. Kempen, D. Bouley, A. Xu, Z. Liu, N.A. Melosh, H. Dai, R. Sinclair, S.S. Gambhir, Shape matters: intravital microscopy reveals surprising geometrical dependence for nanoparticles in tumor models of extravasation. Nano Lett. 12(7), 3369-3377 (2012). https://doi.org/10.1021/nl204175t 
24. C. Sönnichsen, T. Franzl, T. Wilk, G. Von Plessen, J. Feldmann, Plasmon resonances in large noble-metal clusters. New J. Phys. 4(8), 93-94 (2002). https://doi.org/10.1088/1367-2630/4/1/393

25. P.J. Kempen, A.S. Thakor, C. Zavaleta, S.S. Gambhir, R. Sinclair, A scanning transmission electron microscopy approach to analyzing large volumes of tissue to detect nanoparticles. Microsc. Microanal. 19(5), 1290-1297 (2013). https://doi.org/10.1017/ S143192761300192X

26. P.J. Kempen, M.F. Kircher, A. de la Zerda, C.L. Zavaleta, J.V. Jokerst, I.K. Mellinghoff, S.S. Gambhir, R. Sinclair, A correlative optical microscopy and scanning electron microscopy approach to locating nanoparticles in brain tumors. Micron 68, 70-76 (2015). https://doi.org/10.1016/j.micron.2014.09.004

27. A.L. Koh, C.M. Shachaf, S. Elchuri, G.P. Nolan, R. Sinclair, Electron microscopy localization and characterization of functionalized composite organic-inorganic SERS nanoparticles on leukemia cells. Ultramicroscopy 109(1), 111-121 (2008). https:// doi.org/10.1016/j.ultramic.2008.09.004

28. A.S. Thakor, R. Luong, R. Paulmurugan, F.I. Lin, P.J. Kempen, C. Zavaleta, P. Chu, T.F. Massoud, R. Sinclair, S.S. Gambhir, The fate and toxicity of Raman-active silica-gold nanoparticles in mice. Sci. Transl. Med. 3(79), 79ra33 (2011)

29. P.J. Kempen, Electron microscopy investigation of nanoparticles conjugated to biological systems. Ph.D. thesis, Stanford University (2012)

30. S.A. Maier, Localized surface plasmons, in Plasmonics: Fundamentals and Applications (Springer US, New York, NY, 2007), pp. 65-88

31. P.L. Stiles, J.A. Dieringer, N.C. Shah, R.P. Van Duyne, Surfaceenhanced Raman spectroscopy. Annu. Rev. Anal. Chem. 1, 601-626 (2008)

32. J. Nelayah, M. Kociak, O. Stéphan, F.J.G. De Abajo, M. Tencé, L. Henrard, D. Taverna, I. Pastoriza-Santos, L.M. Liz-Marzán, C. Colliex, Mapping surface plasmons on a single metallic nanoparticle. Nat. Phys. 3(5), 348-353 (2007). https://doi.org/10.1038/ nphys 575

33. E.P. Bellido, A. Manjavacas, Y. Zhang, Y. Cao, P. Nordlander, G.A. Botton, Electron energy-loss spectroscopy of multipolar edge and cavity modes in silver nanosquares. ACS Photonics 3(3), 428-433 (2016)

34. E.P. Bellido, G.D. Bernasconi, D. Rossouw, J. Butet, O.J. Martin, G.A. Botton, Self-similarity of plasmon edge modes on Koch fractal antennas. ACS Nano 11(11), 11240-11249 (2017). https:// doi.org/10.1021/acsnano.7b05554

35. M. Bosman, V.J. Keast, M. Watanabe, A.I. Maaroof, M.B. Cortie, Mapping surface plasmons at the nanometre scale with an electron beam. Nanotechnology 18(16), 165505 (2007)

36. A.L. Koh, A.I. Fernández-Domínguez, D.W. McComb, S.A. Maier, J.K. Yang, High-resolution mapping of electron-beamexcited plasmon modes in lithographically defined gold nanostructures. Nano Lett. 11(3), 1323-1330 (2011). https:// doi.org/10.1021/nl104410t

37. J.A. Scholl, A.L. Koh, J.A. Dionne, Quantum plasmon resonances of individual metallic nanoparticles. Nature 483(7390), 421-427 (2012)

38. S.J. Madsen, M. Esfandyarpour, M.L. Brongersma, R. Sinclair, Observing plasmon damping due to adhesion layers in gold nanostructures using electron energy loss spectroscopy. ACS Photonics 4(2), 268-274 (2017). https://doi.org/10.1021/acsph otonics.6b00525

39. M.M. Miller, A.A. Lazarides, Sensitivity of metal nanoparticle surface plasmon resonance to the dielectric environment. J. Phys. Chem. B 109(46), 21556-21565 (2005). https://doi.org/ 10.1021/jp054227y

40. A.D. McFarland, M.A. Young, J.A. Dieringer, R.P. Van Duyne, Wavelength-scanned surface-enhanced Raman excitation spectroscopy. J. Phys. Chem. B 109(22), 11279-11285 (2005). https://doi.org/10.1021/jp050508u

41. Y. Tian, Z. Shuai, J. Shen, L. Zhang, S. Chen, C. Song, B. Zhao, Q. Fan, L. Wang, Plasmonic heterodimers with binding sitedependent hot spot for surface-enhanced Raman scattering. Small 14(24), 1800669 (2018)

42. K.D. Tsuei, E.W. Plummer, A. Liebsch, K. Kempa, P. Bakshi, Multipole plasmon modes at a metal surface. Phys. Rev. Lett. 64(1), 44-47 (1990). https://doi.org/10.1103/PhysRevLett.64.44

43. A.L. Koh, K. Bao, I. Khan, W.E. Smith, G. Kothleitner, P. Nordlander, S.A. Maier, D.W. McComb, Electron energy-loss spectroscopy (EELS) of surface plasmons in single silver nanoparticles and dimers: influence of beam damage and mapping of dark modes. ACS Nano 3(10), 3015-3022 (2009)

44. M.W. Chu, V. Myroshnychenko, C.H. Chen, J.P. Deng, C.Y. Niou, F.J.G. De Abajo, Probing bright and dark surface-plasmon modes in Individual and coupled noble metal nanoparticles using an electron beam. Nano Lett. 9(1), 399-404 (2009). https://doi.org/ $10.1021 / \mathrm{nl} 803270 \mathrm{x}$

45. U. Hohenester, H. Ditlbacher, J.R. Krenn, Electron-energy-loss spectra of plasmonic nanoparticles. Phys. Rev. Lett. 103(10), 106801 (2009)

46. Y. Zeng, S.J. Madsen, A.B. Yankovich, E. Olsson, R. Sinclair, Comparative electron and photon excitation of localized surface plasmon resonance in lithographic arrays for enhanced Raman scattering. Nanoscale 12, 23768-23779 (2020)

47. I. Alber, W. Sigle, S. Muller, R. Neumann, O. Picht, M. Rauber, P.A. Van Aken, M.E. Toimil-Molares, Visualization of multipolar longitudinal and transversal surface plasmon modes in nanowire dimers. ACS Nano 5(12), 9845-9853 (2011)

48. X. Fan, W. Zheng, D.J. Singh, Light scattering and surface plasmons on small spherical particles. Light 3(6), e179 (2014)

49. J. Liu, F. Erogbogbo, K.T. Yong, L. Ye, J. Liu, R. Hu, H. Chen, Y. Hu, Y. Yang, J. Yang, I. Roy, N.A. Karker, M.T. Swihart, P.N. 
Prasad, Assessing clinical prospects of silicon quantum dots: Studies in mice and monkeys. ACS Nano 7(8), 7303-7310 (2013). https://doi.org/10.1021/nn4029234

50. V. Křápek, A. Konečná, M. Horák, F. Ligmajer, M. Stöger-Pollach, M. Hrtoň, J. Babocký, T. Šikola, Independent engineering of individual plasmon modes in plasmonic dimers with conductive and capacitive coupling. Nanophotonics 9(3), 623-632 (2019)

51. W. Yang, Y.F. Chou Chau, S.C. Jheng, Analysis of transmittance properties of surface plasmon modes on periodic solid/outline bowtie nanoantenna arrays. Phys. Plasmas 20(6), 064503 (2013). https://doi.org/10.1063/1.4804405

52. P. Zhan, T. Wen, Z.G. Wang, Y. He, J. Shi, T. Wang, X. Liu, G. Lu, B. Ding, DNA origami directed assembly of gold bowtie nanoantennas for single-molecule surface-enhanced Raman scattering. Angew. Chem. 57(11), 2846-2850 (2018). https://doi.org/10. 1002/anie.201712749

53. A.B. Yankovich, R. Verre, E. Olsen, A.E.O. Persson, V. Trinh, G. Dovner, M. Kall, E. Olsson, Multidimensional hybridization of dark surface plasmons. ACS Nano 11(4), 4265-4274 (2017)

54. M. Hrtoň, A. Konečná, M. Horák, T. Šikola, V. Křápek, Plasmonic antennas with electric, magnetic, and electromagnetic hot spots based on Babinet's principle. Phys. Rev. Appl. 13(5), 054045 (2020). https://doi.org/10.1103/PhysRevApplied.13.054045

55. J.J. Mock, M. Barbic, D.R. Smith, D.A. Schultz, S. Schultz, Shape effects in plasmon resonance of individual colloidal silver nanoparticles. J. Chem. Phys. 116(15), 6755-6759 (2002)
56. N. Hoshyar, S. Gray, H. Han, G. Bao, The effect of nanoparticle size on in vivo pharmacokinetics and cellular interaction. Nanomedicine 11(6), 673-692 (2016). https://doi.org/10.2217/nnm. 16.5

57. L. Rodríguez-Lorenzo, Z. Krpetic, S. Barbosa, R.A. AlvarezPuebla, L.M. Liz-Marzán, I.A. Prior, M. Brust, Intracellular mapping with SERS-encoded gold nanostars. Integr. Biol. 3(9), 922-926 (2011). https://doi.org/10.1039/c1ib00029b

58. H. Arami, S. Kananian, R. Khalifehzadeh, C.B. Patel, E. Chang, Y. Tanabe, Y. Zeng, S.J. Madsen, M. Mandella, M. Ferro, R. Sinclair, Z. Bao, A.Y. Poon, S.S. Gambhir, A remotely-controlled bioelectronic system for cancer treatment. Science 1, 1 (2021)

59. J. Liu, C. Detrembleur, M.C. De Pauw-Gillet, S. Mornet, C. Jérôme, E. Duguet, Gold nanorods coated with mesoporous silica shell as drug delivery system for remote near infrared light-activated release and potential phototherapy. Small 11(19), 2323-2332 (2015). https://doi.org/10.1002/smll.201402145

60. X. Fu, Y. Wang, Y. Liu, H. Liu, L. Fu, J. Wen, J. Li, P. Wei, L. Chen, A graphene oxide/gold nanoparticle-based amplification method for SERS immunoassay of cardiac troponin I. Analyst 144(5), 1582-1589 (2019)

61. H.K. Lee, Y.H. Lee, C.S.L. Koh, G.C. Phan-Quang, X. Han, C.L. Lay, H.Y.F. Sim, Y.C. Kao, Q. An, X.Y. Ling, Designing surfaceenhanced Raman scattering (SERS) platforms beyond hotspot engineering: Emerging opportunities in analyte manipulations and hybrid materials. Chem. Soc. Rev. 48(3), 731-756 (2019) 